\title{
Signos y síntomas asociados a trastornos temporomandibulares en niños y adolescentes. Cali, Colombia
}

\section{Signs and symptoms associates of temporomandibular disorders in children and adolescents. Cali, Colombia}

\author{
Libia Soto Llanos ${ }^{1}$, , Carolina Rodríguez-Manjarrés ${ }^{2}$, Francia Elena Triana-Escobar ${ }^{3}$, \\ Ángela María Duque-Borrero 4 (i)
}

Tipología: Artículo de investigación científica y tecnológica

Para citar este artículo: Soto-Llanos L, Rodríguez-Manjarrés C, Triana-Escobar FE, Duque-Borrero AM. Signos y síntomas asociados a trastornos temporomandibulares en niños y adolescentes. Cali, Colombia. Duazary. 2019 septiembre; 16(3): 54 - 62. Doi: http://dx.doi.org/10.21676/2389783X.2968

Recibido en junio 25 de 2018

Aceptado en marzo 15 de 2019

Publicado en línea en septiembre 01 de 2019

\section{RESUMEN}

Los trastornos temporomandibulares (TTM) en pacientes pediátricos se han estudiado poco. La necesidad de realizar un diagnóstico oportuno hace que exista mayor interés por parte de los investigadores para orientar a los odontólogos. El objetivo del presente estudio fue identificar la presencia de trastornos temporomandibulares registrados en las historias clínicas de pacientes atendidos en la Escuela de Odontopediatría de la Universidad del Valle (Cali, Colombia). Se realizó un estudio observacional descriptivo y transversal para evaluar presencia de trastornos temporomandibulares en pacientes, en edades entre cuatro y 14 años, que asistieron a las clínicas de dicha escuela durante 2013 y 2016 . Se revisaron 712 historias; $56 \%$ de sexo masculino y $44 \%$ de sexo femenino. Los signos y síntomas asociados con los TTM más frecuentes fueron el clic bilateral $(36,4 \%)$, la desviación y deflexión mandibular $(27,1 \%)$ y el dolor a la palpación $(24,0 \%)$, mientras que la subluxación $(1,6 \%)$ y el chasquido articular en apertura $(0,8 \%)$ fueron los de menor presencia. Se concluyó que los trastornos de la articulación temporomandibular (ATM) cada vez son mayores. Es importante, por tanto, realizar investigaciones que involucren los factores de riesgo y/o desencadenantes, la intervención oportuna y el manejo multidisciplinario de esta alteración.

Palabras clave: adolescentes; niños; trastornos; articulación temporomandibular.

1. Universidad del Valle. Cali, Colombia. Correo: libia.soto@ correounivalle.edu.co - https://orcid.org/0000-0002-32527558

2. Universidad del Valle. Cali, Colombia. Correo: carordgz86@ hotmail.com - https://orcid.org/0000-0003-4328-0156

3. Universidad del Valle. Cali, Colombia. Correo: franciaelenatriana@ hotmail.com - https://orcid.org/0000-0002-2784-9106 4. Universidad del Valle. Cali, Colombia. Correo: angela.duque@ correounivalle.edu.com - https://orcid.org/0000-00028826-0661 


\section{ABSTRACT}

Temporomandibular joint disorders in children and adolescents have been studied infrequently. The need for a timely diagnosis makes it more interesting for researchers to perform studies that advise dentists. The objective of the study was to identify the frequency of mandibular disorders in the clinical records of children and adolescents treated at the Pediatric Dentistry Clinic of the Universidad Del Valle. A descriptive and cross-sectional observational study was carried out to evaluate the prevalence of temporomandibular disorders in patients in aged 4 to 14 years attending in the Pediatric Dentistry Clinics of the University of Valle (Cali-Colombia) during January 2013 and June 2016. 712 stories were reviewed; 397 (56\%) were boys and 315 (44\%) girls. 129 reports of the presence of some type of TMJ level, compatible with $18.11 \%$ of the sample studied. The most prevalent signs were bilateral click (36.4\%), mandibular deviation and deflection (27.1\%) and pain on palpation (24.0\%). In contrast, subluxation (1.6\%) and the jump in ATM at opening $(0.8 \%)$ were the minors. It is concluded that disorders increasing daily, it is necessary to carry out investigations involving risk factors and / or triggers, timely or early intervention and the multidisciplinary management that this affectation requires.

Keywords: adolescents; children; disorders; temporomandibular joint.

\section{INTRODUCCIÓN}

Los trastornos temporomandibulares (TTM) se definen como una alteración funcional del sistema masticatorio que involucra un grupo de diagnósticos musculares y articulares específicos tales como quejas de dolor en la región de la articulación temporomandibular (ATM), fatiga de los músculos faciales de la cervicomielitis (especialmente músculos masticatorios), limitación del movimiento mandibular y la presencia de chasquidos articulares $^{1,2}$. La etiología es multifactorial y, por lo tanto, difícil de establecer; se puede relacionar con diferentes factores de tipo funcional, estructural o hereditario y otros fisiológicos, psicológicos y/o sociales, entre los que se incluyen lesiones traumáticas, enfermedades sistémicas inmunomediadas, crecimientos neoplásicos, estrés emocional, interferencias oclusales, mala posición o pérdida de dientes, cambios posturales, disfunciones de la musculatura masticatoria y estructuras adyacentes, cambios extrínsecos e intrínsecos de la estructura de la ATM, movimientos no funcionales de la mandíbula (bruxismo), hábitos de apretamiento de dientes o una combinación de tales factores ${ }^{3-5}$.

Los estudios de prevalencia reportan que los TTM se han comenzado a diagnosticar desde la infancia. Estos trastornos son desarrollados en la adolescencia, y en la adultez llegan a ser severos.
Algunos autores reportan incluso una asociación del crecimiento puberal con el incremento de la prevalencia de $\mathrm{TTM}^{6}$, la cual oscila entre el $20 \%$ y el 50\%. Ahora, si bien puede afirmarse que esta condición afecta más a los adultos, hay estudios que presentan prevalencia de signos y síntomas en un rango del $16 \%$ a $68 \%$ en niños y adolescentes. La variabilidad en la prevalencia se puede atribuir la población estudiada y al método de evaluación empleado para determinar la presencia de los trastornos temporomandibulares, ya que no existe un único consenso en los criterios de diagnóstico o en el uso de instrumentos válidos que sean exclusivos para realizar este análisis ${ }^{7-9}$.

Los criterios empleados en la gran mayoría de los estudios de prevalencia para diagnosticar TTM varían entre el índice de Helkimo, el protocolo de criterios diagnósticos para desórdenes temporomandibulares (RDC/TMD por sus siglas en inglés), el índice craneomandibular y los cuestionarios anamnésicos $^{8,10}$. Es claro entonces que se necesitan métodos apropiados y estandarizados para identificar la presencia de TTM, lo que permitiría mejorar y comprender los aspectos patológicos a fin de abordarlos de forma oportuna y preventiva ${ }^{11}$. En particular, el RDC/TMD basado en la evidencia es recomendado para entornos clínicos y de investigación. Este instrumento validado permite la identificación de pacientes con una 
gama de presentaciones de TMD simples y complejas $^{12}$. Sin embargo, en muchas instituciones aún se usan instrumentos de evaluación cortos y simples.

El objetivo de este estudio fue identificar la prevalencia de signos y síntomas asociados a TTM registrados en las historias clínicas de niños y adolescentes de cuatro a 14 años atendidos en la Escuela de Odontología de la Universidad del Valle (Cali, Colombia) durante el período comprendido entre enero de 2013 y junio de 2016.

\section{MATERIALES Y MÉTODOS}

\section{Tipo de estudio}

Estudio de tipo observacional descriptivo y transversal para evaluar la presencia de signos y síntomas asociados a los TTM en niños y adolescentes en edades entre los cuatro y 14 años que asistieron a las clínicas de odontopediatría de pregrado y posgrado de la Escuela de Odontología de la Universidad del Valle en la ciudad de Cali, Colombia, entre enero de 2013 y junio de 2016.

\section{Población y muestra}

La población del estudio consistió en la revisión de 749 historias clínicas de pacientes que asistieron a consulta de odontopediatría entre enero de 2013 y junio de 2016. Estas historias fueron revisadas por cuatro investigadores, verificando los criterios de inclusión y exclusión.

\section{Criterios de inclusión}

Se incluyeron las historias clínicas, con diligenciamiento completo, de pacientes con edad entre cuatro y 14 años que estuvieran sistémicamente sanos, con exámenes de protocolo clínico anexos de oclusión y ATM. Los signos y síntomas de TTM registrados en las historias clínicas de la Escuela de Odontología de la Universidad del Valle entre 2013 y 2016 fueron los siguientes: sonidos de la articulación temporomandibular, desviación y deflexión mandibular, subluxación, dolor a la palpación en el área articular, ruido articular, limitación en apertura, hipertrofia muscular y chasquido articular en apertura. Hasta la fecha en que se realizó la revisión de historias clínicas no se había implementado un protocolo de criterios de diagnóstico de investigación estandarizado para TTM.

\section{Criterios de exclusión}

Historias clínicas que no estuvieran completamente diligenciadas, de pacientes con edades por fuera del rango de cuatro a 14 años.

\section{Análisis estadístico}

Los datos sobre la prevalencia de signos y síntomas se analizaron considerando dos grupos según el sexo (femenino o masculino) y la edad en dos rangos: de cuatro a nueve años y de 10 a 14 años. Para cada categoría de signos y síntomas asociados a TTM de los grupos, la prevalencia fue expresada en porcentaje con respecto al número de sujetos de cada grupo, $\mathrm{y}$ los grupos se compararon mediante el análisis de chi $^{2}$. Todos los análisis se realizaron utilizando el software estadístico S.P.S.S. 24 para Windows, y todas las pruebas estadísticas se establecieron a un nivel de confianza del $95 \%$ $(\mathrm{p}<0,05)$.

\section{Declaración sobre aspectos éticos}

Para la realización del estudio se contó con el aval del director de la Escuela de Odontología de la Universidad del Valle, en Cali, Colombia, para acceder a la información registrada en las historias clínicas de los pacientes que asistieron a las clínicas de odontopediatría de dicha universidad. Para esta investigación se tuvieron en cuenta la Resolución 8430 de 1993 sobre investigaciones en salud, la Ley 1273 de 2009 sobre la protección de la información y de los datos y la Ley 1266 de 2009 de habeas data, garantizando confidencialidad de la información con fines de vigilancia en salud pública y demás entidades que participaron en el proceso. Además, se pudo identificar que se respetó lo establecido en la Declaración de Helsinki de la Asociación Médica Mundial, en donde se 
relacionan los principios éticos para las investigaciones médicas en seres humanos.

Es importante relacionar esta investigación, en particular, con el artículo 11 de la Resolución 8430 de 1993 emanada por el Ministerio de Salud, cuyo literal a identifica investigaciones sin riesgo, dado que el presente es un estudio que empleó técnicas y métodos de investigación documental retrospectivos y no se realizó ninguna intervención o modificación intencionada de las variables biológicas, fisiológicas, psicológicas o sociales de los individuos que participaron. Se especifica en el presente estudio que la fuente primaria fue la revisión de historias clínicas de los pacientes.

\section{RESULTADOS}

Se revisaron 749 historias, de las cuales 129 cumplieron los criterios de inclusión. Los datos demográficos de sexo presentaron una frecuencia similar, con $51,2 \%$ de niñas y $48,8 \%$ de niños que están entre los cuatro y los nueve años de edad, y $66,7 \%$ y $33,3 \%$ respectivamente para el grupo de 10 a 14 años (Tabla 1).

Con la información obtenida de los signos y síntomas asociados a TTM se calculó la prevalencia en sujetos que estaban en un rango de edad de cuatro a nueve años, quienes representaron el $66,7 \%$ de la muestra, y en los del grupo de 10 a 14 años, equivalentes al 33,3\%. Así se encontró que la relación entre la prevalencia de signos y síntomas asociados a TTM y la edad no presentó una diferencia estadísticamente significativa $(\mathrm{p}=0,07)$. Entre los sujetos más jóvenes se registró en mayor proporción el clic bilateral $(29,5 \%)$, mientras que entre los sujetos mayores este signo correspondió al 7,0\% de los casos (Tabla 2).

Tabla 1. Datos demográficos de 129 niños/adolescentes de una población que asistió a la clínica de odontopediatría de la Escuela de Odontología de la Universidad del Valle, Cali, Colombia.

\begin{tabular}{|l|c|c|c|}
\hline \multicolumn{1}{|c|}{ Edad } & \multicolumn{1}{|c|}{$\begin{array}{c}\text { Niña } \\
\mathbf{n = 6 6}\end{array}$} & $\begin{array}{c}\text { Niño } \\
\mathbf{n = 6 3}\end{array}$ & $\begin{array}{c}\text { Total } \\
\mathbf{n = 1 2 9}\end{array}$ \\
\hline 4-9 años & $43(33,3)$ & $43(33,3)$ & $86(66,7)$ \\
\hline 10-14 años & $23(17,8)$ & $20(15,5)$ & $43(33,3)$ \\
\hline Total & $66(51,2)$ & $63(48,8)$ & $129(100)$ \\
\hline
\end{tabular}

Las cifras muestran el número de sujetos y porcentaje n $(\%)$.

Tabla 2. Prevalencia de signos y síntomas asociados al TTM por rango de edad (n=129)

\begin{tabular}{|l|c|c|}
\hline \multicolumn{1}{|c|}{ Signos y síntomas } & $\begin{array}{c}\mathbf{4 - 9} \text { años } \\
\mathbf{n = 8 6}\end{array}$ & $\begin{array}{c}\mathbf{1 0 - 1 4} \text { años } \\
\mathbf{n = 4 3}\end{array}$ \\
\hline & \multicolumn{2}{|c|}{$p=0,07$} \\
\hline Clic bilateral & $38(29,5)$ & $9(7,0)$ \\
\hline Desviación y deflexión mandibular & $20(15,5)$ & $15(11,6)$ \\
\hline Subluxación & $2(1,6)$ & $0(0,0)$ \\
\hline Dolor a la palpación & $16(12,4)$ & $3(11,6)$ \\
\hline Ruido articular & $3(2,3)$ & $0(0,0)$ \\
\hline Limitación en apertura & $4(3,1)$ & $1(0,8)$ \\
\hline Hipertrofia muscular & $2(1,6)$ & $0(0,0)$ \\
\hline Chasquido articular (apertura) & $1(0,8)$ & \\
\hline
\end{tabular}

Las cifras muestran el número de sujetos y porcentaje n (\%). 
La prevalencia de signos y síntomas también se analizó entre los grupos de niñas y niños. Los resultados no mostraron diferencia estadísticamente significativa entre ellos $(\mathrm{p}=476)$; de hecho, se encontró una frecuencia muy similar entre los grupos para la presencia de signos y síntomas, a excepción de la desviación y deflexión mandibular, que se presentó en el $17,1 \%$ de las niñas y en el $10,1 \%$ de los niños, siendo el segundo más frecuente entre los signos y síntomas asociados al TTM (Tabla 3).

Tabla 3. Prevalencia de signos y síntomas asociados con los TTM por sexo $(n=129)$

\begin{tabular}{|l|c|c|c|}
\hline \multicolumn{1}{|c|}{ Signos y síntomas } & $\begin{array}{c}\text { Niñas } \\
\mathbf{n = 6 6}\end{array}$ & $\begin{array}{c}\text { Niños } \\
\mathbf{n = 6 3}\end{array}$ & Total \\
\hline & \multicolumn{2}{|c|}{$p=476$} & \\
\hline Clic bilateral & $23(17,8)$ & $24(18,6)$ & $47(36,4)$ \\
\hline Desviación y deflexión mandibular & $22(17,1)$ & $13(10,1)$ & $35(27,1)$ \\
\hline Subluxación & $1(0,8)$ & $1(0,8)$ & $2(1,6)$ \\
\hline Dolor a la palpación & $14(10,9)$ & $17(13,2)$ & $31(24,0)$ \\
\hline Ruido articular & $3(2,3)$ & $3(2,3)$ & $6(4,7)$ \\
\hline Limitación en apertura & $2(1,6)$ & $2(1,6)$ & $4(3,1)$ \\
\hline Hipertrofia muscular & $0(0,0)$ & $3(2,3)$ & $3(2,3)$ \\
\hline chasquido articular (apertura) & $1(0,8)$ & $0(0,0)$ & $1(0,8)$ \\
\hline
\end{tabular}

Las cifras muestran el número de sujetos y porcentaje $\mathrm{n}(\%)$.

\section{DISCUSIÓN}

Los diferentes estudios realizados han mostrado que el sexo femenino es el que presenta una mayor prevalencia de signos y síntomas asociados con los $\mathrm{TTM}^{13}$, atribuidos a factores hormonales, posturales, emocionales, oclusales y funcionales, así como a la predisposición genética $^{14,15}$. Estas diferencias ligadas al sexo femenino se resumen como causa de factores neuropsicológicos y psicosociales, pues aparentemente las mujeres poseen menor umbral del dolor y son más vulnerables al estrés ${ }^{16,17}$, lo que también se ha reportado en la población de adolescente $^{18}$. Estos datos coinciden con los resultados obtenidos en la presente investigación, donde las niñas se vieron ligeramente más afectadas que los niños con un $51,2 \%$ y $48,8 \%$ respectivamente.

Se ha encontrado también que las condiciones de TTM en la edad prepuberal son principalmente de origen muscular y luego, con la edad, se complementan con trastornos intracapsulares ${ }^{7,19}$. Estos resultados se relacionan con lo observado en la investigación en la Escuela de Odontología de Cali, donde los signos con mayor frecuencia fueron el clic bilateral $(36,4 \%)$, la desviación y deflexión mandibular $(27,1 \%)$ y el dolor a la palpación $(24,0 \%)$. Por el contrario, la subluxación, que se presentó en un 1,6\%, y el chasquido articular en apertura, con 0,8\%, fueron los de menor frecuencia. En cuanto a síntomas, se ha reportado que el dolor de cabeza es el más frecuente, seguido del ruido articular ${ }^{20}$. Gran parte de los clics observados en niños y adolescentes es temporal, con una incidencia significativamente mayor de clics en el sexo femenino $^{21}$.

Los estudios realizados en niños y adolescentes reportan que, dependiendo de la etapa de crecimiento y desarrollo en que se presente la alteración, estos sujetos pueden tener un porcentaje más elevado de diagnósticos específicos del TTM. Los músculos orofaciales de los individuos jóvenes tienen una mayor capacidad de adaptación fisiológica durante el crecimiento y el desarrollo, lo que a su vez podría minimizar los síntomas de TTM; esto significa que los niños se acostumbran al dolor y lo consideran un sentimiento normal ${ }^{7,10}$. Con esto en mente, cabe anotar que en la presente 
investigación había un 50\% más de niños y niñas entre cuatro y nueve años que de 10 a 14 años, lo que puede influir en los resultados, encontrando mayor frecuencia de signos y síntomas asociados al TTM en este grupo más joven. Estudios en adolescentes reportan que los diagnósticos específicos de los trastornos craneomandibulares eran frecuentes aunque generalmente leves: específicamente, dolor a la palpación muscular, seguido de la desviación de apertura ${ }^{22}$.

La variación en el rango de edad es un factor que determina los hallazgos de las investigaciones. Aquellas que involucran grupos de estudio de pacientes en los tres estadios de la dentición reportan una mayor prevalencia de signos y síntomas asociados al TTM entre los 12 y los 14 años $^{23}$, aunque algunos autores ${ }^{24,25}$ plantean que estos pueden aparecer a cualquier edad, ya sea en pacientes jóvenes o en adultos. En el caso del presente estudio, si bien se incluyó un rango de edad que correspondía a niños y adolescentes, los análisis compararon dos rangos de edad, separando en lo posible estos dos grupos etarios para disminuir el riesgo de sesgo en los hallazgos, lo que llevó a identificar diferencias estadísticamente significativas en términos de prevalencia.

Una limitación de este trabajo fue la fuente de información debido a que el instrumento de evaluación con el que se reportan los signos y síntomas en las historias clínicas de los pacientes es un cuestionario corto no estandarizado de acuerdo a los criterios diagnósticos para desórdenes temporomandibulares como el índice de Helkimo, el índice craneomandibular, los cuestionarios anamnésicos ${ }^{8,10}$ o el DC/TMD ${ }^{12}$. Por esta razón se recomienda, para futuras investigaciones y para la práctica clínica universitaria, usar métodos estandarizados para identificar la presencia de TTM, lo que permitiría mejorar la evaluación y diagnóstico de los pacientes en un estado temprano, comprendiendo integralmente la patología. Esto llevará a su vez a mayores investigaciones de los aspectos asociados al TTM, su diagnóstico y prevención.

\section{CONCLUSIÓN}

En vista de que la presencia de los TTM cada día va en aumento, es necesario realizar investigaciones que involucren los factores de riesgo y/o desencadenantes, la intervención oportuna o temprana y el manejo multidisciplinario que esta requiere para brindar la solución a las necesidades de la población afectada, en especial para casos de niños y adolescentes.

También es importante realizar estudios que evalúen la frecuencia de los TTM en niños y adolescentes empleando los criterios diagnósticos de los trastornos temporomandibulares para obtener resultados precisos. De este modo será posible unificar conceptos y plantear protocolos de manejo de los TTM en la población pediátrica, y fortalecer áreas como el diagnóstico, el pronóstico y los planes de tratamiento dirigidos a la prevención de esta patología.

\section{DECLARACIÓN SOBRE CONFLICTOS DE INTERES}

Los autores declaran no tener conflicto de intereses en este artículo.

\section{CONTRIBUCIÓN DE LOS AUTORES}

Primer autor: diseño del trabajo original, adquisición de datos para el trabajo, redacción del manuscrito, revisión de precisión del manuscrito, aprobación definitiva de la versión por publicar.

Segundo autor: diseño del trabajo original, análisis de datos para el trabajo, revisión crítica de contenido intelectual, aprobación definitiva de la versión por publicar, revisión de integridad del manuscrito.

Tercer autor: desarrollo de la idea presentada, búsqueda de la teoría, interpretación de datos para el trabajo para el trabajo, redacción del manuscrito, aprobación definitiva de la versión 
Libia Soto Llanos, Carolina Rodríguez-Manjarrés, Francia Elena Triana-Escobar, Ángela María Duque-Borrero

por publicar, revisión de integridad del manuscrito.

Cuarto autor: desarrollo de la idea presentada, búsqueda de la teoría, interpretación de datos para el trabajo para el trabajo; revisión crítica de contenido intelectual, revisión de precisión del manuscrito, aprobación definitiva de la versión por publicar.

\section{REFERENCIAS BIBLIOGRÁFICAS}

1. Rokaya D, Suttagul K, Joshi S, Bhattarai BP, Shah PK, Dixit S. An epidemiological study on the prevalence of temporomandibular disorder and associated history and problems in Nepalese subjects. J Dent Anesth Pain Med. 2018 Feb;18(1):27-33. Doi: http://dx.doi.org/10.17245/jdapm.2018.18.1.27

2. Manfredini D, Lobbezoo F. Relationship between bruxism and temporomandibular disorders: a systematic review of literature from 1998 to. Oral Surg Oral Med Oral Pathol Oral Radiol Endod. 2010 Jun;109(6):e26-e50. Doi: http://dx.doi.org/10.1016/j.tripleo.2010.02.013

3. Howard JA. Temporomandibular Joint Disordersin Children. Dent Clin NA. 2013 Jan;57(1):99-127.

Doi: http://dx.doi.org/10.1016/j.cden.2012.10.001.

4. De Kanter RJAM, Battistuzzi PGFCM, Truin G-J. Temporomandibular Disorders: "Occlusion" Matters! Pain Res Manag. 2018;2018(8746858):1-13. Doi: http://dx.doi.org/10.1155/2018/8746858

5. Conti PC, Ferreira PM, Pegoraro LF, Conti J V, Salvador MC. A cross-sectional study of prevalence and etiology of signs and symptoms of temporomandibular disorders in high school and university students. J Orofac Pain. 1996 Summer;10(3):254-262.

6. Marpaung C, Lobbezoo F, Selms MKA Van. Temporomandibular Disorders among
Dutch Adolescents : Prevalence and Biological, Psychological , and Social Risk Indicators. Pain Res Manag. 2018;2018(5053709):1-9. Doi: http://dx.doi.org/10.1155/2018/5053709

7. Al-khotani A, Naimi-akbar A, Albadawi E, Ernberg M, Hedenberg-magnusson B, Christidis N. Prevalence of diagnosed temporomandibular disorders among Saudi Arabian children and adolescents. J Headache Pain. $2016 \quad$ Apr;17(41):2-11. Doi: http://dx.doi.org/10.1186/s10194-016-0642-9

8. Tecco S, Crincoli V, Bisceglie B, Saccucci M, Macrí M, Polimeni A, et al. Signs and symptoms of temporomandibular joint disorders in caucasian children and adolescents. Cranio - J Craniomandib Pract. 2011 Feb;29(1):71-79. Doi: http://dx.doi.org/10.1179/crn.2011.010

9. Köhler AA, Helkimo AN, Magnusson T, Hugoson A. Prevalence of symptoms and signs indicative of temporomandibular disorders in children and adolescents. A cross-sectional epidemiological investigation covering two decades. Eur Arch Paediatr Dent. 2009 Nov;10(1):16-25.

10. Lauriti L, Motta LJ, Da Costa PF, LeaL de Godoy CH, Almeida T, Santos K, et al. Are Occlusal Characteristics, Headache, Parafunctional Habits and Clicking Sounds Associated with the Signs and Symptoms of Temporomandibular Disorder in Adolescents? J Phys Ther Sci. 2013;25(10):1331-1334. Doi: http://dx.doi.org/10.1589/jpts.25.1331

11. Allori AC, Chang CC, Farin R, Grayson BH, Warren SM, Mccarthy JG. Current Concepts in Pediatric Temporomandibular Joint Disorders: Part 1. Etiology, Epidemiology, and Classification. Plast Reconstr Surg. 2010 Oct;126(4):1263-1275. Doi: http://dx.doi.org/10.1097/PRS.0b013e3181ebe2 07 
12. Schiffman E, Ohrbach R, Truelove E, Look J, Anderson G, Goulet JP, List T, et al. Diagnostic Criteria for Temporomandibular Disorders (DC/TMD) for Clinical and Research Applications: Recommendations of the International RDC/TMD Consortium Network* and Orofacial Pain Special Interest Group $\dagger$. J Oral Facial Pain Headache. 2014 Winter;28(1):6-27.

Doi: http://dx.doi.org/10.11607/jop.1151

13. Bertoli DP, Bruzamolin CD, Pizzatto E, Losso EM, Brancher JA, Souza JF. Prevalence of diagnosed temporomandibular disorders: A cross-sectional study in Brazilian adolescents. PLoS One. 2018 Feb;13(2):e0192254.

14. Kafas P, Leeson R. Assessment of pain in temporomandibular disorders: the biopsychosocial complexity. Int J Oral Maxillofac Surg. 2006 Feb;35(2):145-149. Doi: http://dx.doi.org/10.1016/j.ijom.2005.04.023

15. Magnusson T, Egermark I, Carlsson GE. A longitudinal epidemiologic study of signs and symptoms of temporomandibular disorders from 15 to 35 years of age. J Orofac Pain. 2000 Fall;14(4):310-319.

16. Sermet EÜ, Kocasarac HD, Elbay M, Kaya C, Uğurluel C, Baydemir C. Temporomandibular disorders and oral parafunction in children living with their parents and children living in institutional protective care: a comparative study. Int Dent J. 2016 Aug;67(1):20-28.

http://dx.doi.org/10.1111/idj.12253

17. Habib SR, Al Rifaiy MQ, Awan KH, Alsaif A, Alshalan A, Altokais Y. Prevalence and severity of temporomandibular disorders among university students in Riyadh. Saudi Dent J. 2015 Jul;27(3):125-130. Doi: http://dx.doi.org/10.1016/j.sdentj.2014.11.009

18. Hongxing L, Astrøm AN, List T, Nilsson IM, Johansson A. Prevalence of temporomandibular disorder pain in Chinese adolescents compared to an age-matched
Swedish population. J Oral Rehabil. 2016 Apr;43(4):241-248.

Doi: http://dx.doi.org/10.1111/joor.12366

19. Huhtela OS, Student F, Service H, et al. Self-Reported Bruxism and Symptoms of Temporomandibular Disorders in Finnish University Students. J Oral Facial Pain Headache. 2016 Fall;30(4):311-317. Doi: http://dx.doi.org/10.11607/ofph.1674

20. Corsini G, Fuentes R, Bustos L, Borie E, Navarrete A, Navarrete D, et al. Determinación de los Signos y Síntomas de los Trastornos Temporomandibulares, en Estudiantes de 13 a 18 Años de un Colegio de la Comuna de Temuco, Chile. Int J Morphol. 2005;23(4):345352.

21. Torii K. Longitudinal course of temporomandibular joint sounds in Japanese children and adolescents. Head Face Med. 2011 Oct;7:17. Doi: http://dx.doi.org/10.1186/1746$160 \mathrm{X}-7-17$

22. Weiler RM, Santos FM, Kulic MA, Lima MP, Pardini SR, Mori M, et al. International Journal of Pediatric Otorhinolaryngology Prevalence of signs and symptoms of temporomandibular dysfunction in female adolescent athletes and non-athletes. Int $\mathbf{J}$ Pediatr Otorhinolaryngol. 2013 Apr;77(4):519524.

Doi:

http://dx.doi.org/10.1016/j.ijporl.2012.12.024

23. Soto L, Hernández JA, Villavicencio JE. Trastornos de la articulación temporomandibular en escolares de 5 a 14 años de un centro educativo de Cali. Colomb Med. 2001;32(3):116-120.

24. Perlman AE, Lobbezoo F, Zar A, Rubin PF, Selms V, Winocur E. Oral Rehabilitation Self-Reported bruxism and associated factors in Israeli adolescents. J Oral Rehabil. 2016 Mar;43(6):443-450. Doi: http://dx.doi.org/10.1111/joor.12391 
Libia Soto Llanos, Carolina Rodríguez-Manjarrés, Francia Elena Triana-Escobar, Ángela María Duque-Borrero

25. Carlsson GE, Egermark I, Magnusson T. Predictors of signs and symptoms of temporomandibular disorders: a 20-year followup study from childhood to adulthood. Acta
Odontol Scand. 2016 Jul;60(3):180-185. Doi: http://dx.doi.org/10.1080/00016350275374021 4 